\title{
MRI STANDARD TECHNICAL PROTOCOL VERSUS ADVANCED HEPATOBILIARY PROTOCOL IN DIAGNOSING SOLITARY CYSTIC HEPATIC LESIONS
}

\author{
Ameen M. $D^{1}$, Vetriraj $S^{2}$, Girish Venkat $N^{3}$
}

${ }_{1}^{1}$ Associate Professor, Department of Radiodiagnosis.

${ }^{2}$ Associate Professor, Department of Radiodiagnosis.

${ }^{3}$ Assistant Professor, Department of Radiodiagnosis.

\begin{abstract}
BACKGROUND
ABSTRACT

Solitary cystic liver lesions are not uncommon, comes with varying age groups. To decide surgical approach and treatment planning of cystic mass lesion, specific diagnosis with high sensitivity and specificity is required from the clinician's perspective. To approach cystic liver pathology, specific diagnostic MRI protocol algorithm from literature is lacking.

The objective of this study is to illustrate and prove efficacy of MR customised protocol that works better in delineating specific diagnosis of cystic liver lesions as compared to standard protocol approach.
\end{abstract}

\section{MATERIALS AND METHODS}

A descriptive study, sample size of 31 cases were enrolled in the study. Liver biopsy was set as standard reference done prospectively after 2 weeks. Ultrasound proved to be solitary hepatic lesions set as inclusion criteria. Study was done by two different Radiologists blinded with liver biopsy.

\section{RESULTS}

Results were derived by using data descriptive percentage analysis and Chi-square test and Fisher's Exact test. MRI standard protocol specific diagnosis detection of sensitivity, specificity, PPV and NPV are $66.67 \%, 94.44 \%, 66.67 \%$ and $94.44 \%$ as compared to MRI modified approach protocol of $66.67 \%, 100.00 \%, 100.00 \%$ and $94.44 \%$.

\section{CONCLUSION}

MR modified protocol approach for solitary cystic liver lesions proves to have better specificity as compared to standard protocol. In future, prospective clinician should be aware of modified protocol for specific liver pathology for better treatment planning.

\section{KEYWORDS}

Magnetic Resonance Imaging (MRI); Liver Biopsy; Solitary Cystic Liver Lesions.

HOW TO CITE THIS ARTICLE: Ameen MD, Vetriraj S, Venkat GN. MRI standard technical protocol versus advanced hepatobiliary protocol in diagnosing solitary cystic hepatic lesions. J. Evolution Med. Dent. Sci. 2018;7(01):12-15, DOI: 10.14260/jemds/2018/4

\section{BACKGROUND}

The basic functional unit of the liver is the hepatic lobule, which consists of portal triads (hepatic arteriole, portal venule, bile duct, lymphatics and branch of the vagal nerve) at the periphery that surround a central vein (Fig. 1). Hepatic sinusoids (capillaries) connect the portal triad to the central vein and are surrounded by sheets of hepatocytes. Blood flows from the hepatic arterioles and portal venules into the sinusoids, filters across the hepatic lobule and collects in the central vein and hepatic venules to drain into the hepatic veins. Many hepatic tumours are supplied by the hepatic artery and enhance avidly during the late arterial phase in contrast to the described enhancement patterns of other neoplasms, which enhance more avidly in the later phases. $(1,2,3)$

Thus, dynamic multiphasic contrast material-enhanced MR imaging helps to characterise liver lesions.

'Financial or Other Competing Interest': None.

Submission 05-12-2017, Peer Review 16-12-2017,

Acceptance 19-12-2017, Published 01-01-2018.

Corresponding Author:

Dr. Ameen M. D

Flat $1 C$, No. 9

Swaraj Manor Leithe Castle Centre Street,

Santhome, Chennai-600028.

E-mail: drameenmdrd@rocketmail.com

DOI: $10.14260 /$ jemds $/ 2018 / 4$
The liver contains four types of cells: Hepatocytes, Endothelial cells lining the sinusoids, Kupffer cells and Stellate cells. Hepatocytes perform biosynthesis including production of bile, proteins and glucose, and biotransformation of toxins and drugs.(1) Kupffer cells, which are part of the reticuloendothelial system function as macrophages, while stellate cells store fat and vitamin A.(1) Different types of MR imaging contrast media are available that are variably taken up by these cells in the liver and help. However, disadvantages of MR imaging include its longer imaging time and the frequent need for sedation or anaesthesia in smaller children. Cystic hepatic lesions are commonly encountered in daily practice. $(4,3)$ The differential diagnoses range from benign lesions of no clinical significance to malignant and potentially lethal conditions. Many cystic hepatic lesions have classic imaging findings, and the diagnosis can be made with certainty on the basis of imaging alone. In other cases, recognising key radiologic features in combination with reviewing the clinical data usually allows the correct diagnosis. Cystic hepatic lesions can be divided into developmental, inflammatory, neoplastic and trauma-related lesions.(3,5,6) An incidental simple hepatic cyst is the most commonly encountered pathologic finding. The number and morphology of the lesions and determination of whether there is a solid component are the key imaging features that are helpful for approaching the diagnosis of cystic hepatic lesions. 
The pretest probability of a diagnosis is highly affected by the patient's comorbidities and the clinical and laboratory data; thus, imaging studies should be interpreted in the context of the other clinical information for that particular patient. Except simple hepatic cysts and polycystic liver disease which can be confidently diagnosed on the basis of ultrasound only, contrast-enhanced CT or MRI is essential to establish a definitive diagnosis or provide a reasonable differential diagnosis. $(7,8)$

Most of the studies show mixed sensitivity and specificity in solitary cystic liver lesions and specific MR protocol is lacking and not standardised. To the best of our knowledge, proper algorithm approach and specific standardised protocol for solitary cystic liver lesions are emphasised in this study.

\section{MATERIALS AND METHODS}

A descriptive study, sample size of 31 cases were enrolled in the study. Liver biopsy set as reference standard was done prospectively after 2 weeks. Ultrasound proved solitary hepatic lesions studied from January 2016 to August 2017 was set as inclusion criteria. Study was done by two different Radiologists blinded with liver biopsy.

\section{Statistical Analysis}

The collected data were analysed by IBM SPSS Statistics Software 23.0 Version. To describe about the data descriptive statistics frequency analysis, percentage analysis were used. To find the significance in categorical data Chi-square test was used, similarly if the expected cell frequency is less than 5 in $2 \times 2$ tables then the Fisher's Exact was used. To find the significance in categorical data, Chi-Square test and Fisher's Exact test were used. In all the above statistical tools, the probability value of .05 is considered as significant level.

\section{Ethical Clearance}

Written informed consent was obtained from all 31 patients and legally registered. Ethical clearance was also formally obtained from Institutional Board from Annai and Velammal Teaching Hospital.

\section{Image Protocol}

To achieve optimal hepatobiliary imaging, care must be taken to ensure proper patient preparation. Initial patient preparation begins with evaluating the need for sedation or anaesthesia. Typically, children under 6 years of age who are unable to perform a 20-second breath-hold will require sedation or anaesthesia. Patients should take nothing by mouth for 4 hours before the examination. This will help to minimise artefacts from bowel peristalsis and ensure a full gallbladder. To optimally evaluate the vascular system, adequate peripheral venous access is necessary to power inject contrast material.

Conventional $\mathrm{T} 1$ and T2-weighted imaging can be performed with fast spin-echo in coronal and axial planes utilising respiratory-triggered or navigated sequences in at least the axial plane.

Modified protocol MR imaging includes diffusion and ADC axial images. Diffusion-weighted imaging (DWI) has recently become an additional imaging tool to aid in evaluation of hepatic lesions, particularly malignant tumours. Unenhanced MR angiography sequence $(\mathrm{T} 2 *$ images can be obtained on any scanner by performing a gradient-echo sequence multiple times at different TE values using a fixed TR of approximately 150 milliseconds. TE values should typically range from 1 to 20 milliseconds). Image analysis and Interpretation image analysis was done by two experienced radiologists. One radiologist followed protocol of conventional imaging and reported MR cystic hepatic lesion findings and another radiologist reported modified approach protocol and reported cystic hepatic lesion findings.

\section{RESULTS}

Liver biopsy revealed 16 solitary benign cystic liver lesions, 3 malignancies like HCC and hepatoblastoma and 2 patients with atypical cysts with no complication. As Table 1 and Table 3 correlation mentioned above, Table 3 MR modified protocol comparison with biopsy showed better sensitivity and specificity as compared to Table 1 results.

A Routine Protocol and HOE Cross-Tab.

\begin{tabular}{|c|c|c|c|c|}
\hline \multicolumn{2}{|c|}{} & \multicolumn{2}{|c|}{ HPE } & \\
\cline { 2 - 5 } \multicolumn{2}{|c|}{} & M & B or IT & Total \\
\hline \multirow{2}{*}{ ROUTMA } & M & 2 & 1 & 3 \\
\cline { 2 - 5 } & B or IT & 1 & 27 & 28 \\
\hline \multicolumn{2}{|c|}{ Total } & $\mathbf{3}$ & $\mathbf{2 8}$ & $\mathbf{3 1}$ \\
\hline
\end{tabular}

Table 1. Showing Cross Tabulation with Sensitivity Value

\begin{tabular}{|c|c|}
\hline Sensitivity & 66.67 \\
\hline Specificity & 96.43 \\
\hline PPV & 66.67 \\
\hline NPV & 96.43 \\
\hline Accuracy & 81.55 \\
\hline \multicolumn{2}{|c|}{ Table 2. Showing Cross-Tabulation with Sensitivity Value } \\
\hline
\end{tabular}

\begin{tabular}{|c|c|c|c|c|}
\hline & \multicolumn{2}{|c|}{ HPE } & \multirow[b]{2}{*}{ Total } \\
\hline & & $\mathbf{M}$ & B or IT & \\
\hline \multirow[t]{2}{*}{ ADVMR } & $\mathrm{M}$ & 2 & 0 & 2 \\
\hline & B or IT & 1 & 28 & 29 \\
\hline \multicolumn{2}{|c|}{ Total } & 3 & 28 & 31 \\
\hline
\end{tabular}

\begin{tabular}{|c|c|}
\hline Sensitivity & 66.67 \\
\hline Specificity & 100.00 \\
\hline PPV & 100.00 \\
\hline NPV & 96.55 \\
\hline Accuracy & 83.33 \\
\hline Table 4. Mod MR Prot and HPE Cross-Tabulation \\
\hline
\end{tabular}

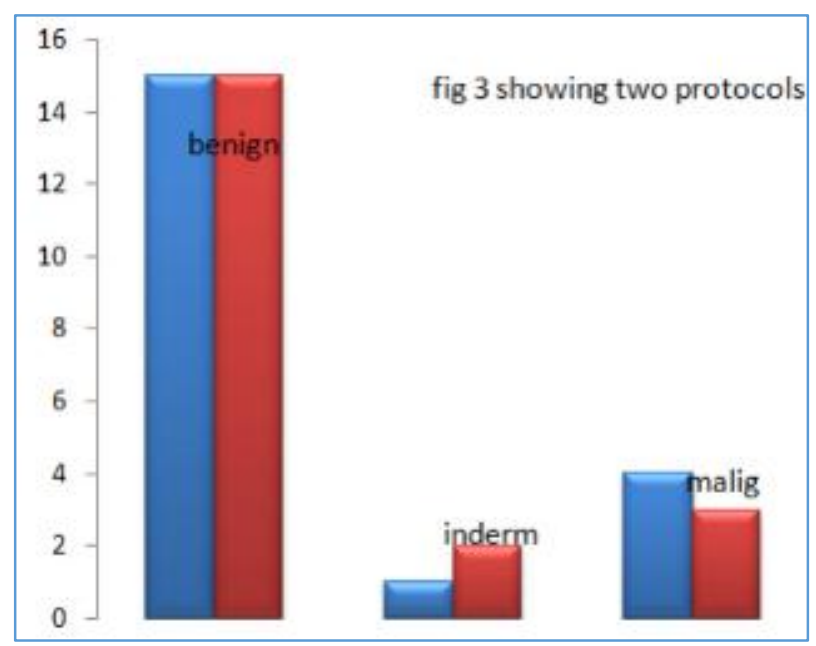


Figure 3 showing Graph analysis of benign cystic liver pathology versus malignant pathology detection by routine approach as compared to liver biopsy findings of atypical cyst and malignant complexity- Routine protocol is not the diagnostic modality of choice.

MRI standard protocol of specific diagnosis detection of sensitivity, specificity, PPV and NPV are 66.67\%, 94.44\%, $66.67 \%$ and $94.44 \%$ as compared to MRI modified approach protocol of $66.67 \%, 100.00 \%, 100.00 \%$ and $94.44 \%$ and accuracy of $84 \%$ as compared to routine MR protocol approach.

\section{DISCUSSION}

Hepatic cysts are typically round or ovoid structures that have an imperceptible wall. These cysts are usually multiple in number and vary in size. The ultrasound features of hepatic cysts are similar to those of simple cysts in other organs. Common features include a well-marginated anechoic structure with enhancement of the posterior wall and increased through transmission (Fig. 1).

On CT and MRI, simple cysts have attenuation (0 - $15 \mathrm{HU})$ and signal intensity (T1 hypointensity, T2 hyperintensity) similar to water. Simple cysts do not show enhancement. Hepatic cysts can rarely become complex as a result of haemorrhage or superinfection; sequelae include development of internal septations, rim calcification and increased attenuation or heterogeneous signal intensity.(8)

Intrahepatic pseudoaneurysm is a rare entity, that is usually a delayed complication of trauma or can be caused by iatrogenic injuries from prior surgery or percutaneous procedures. Pseudoaneurysms appear cystic on ultrasound and on unenhanced CT. The vascular nature of these lesions can be easily established on colour and spectral Doppler imaging. Contrast-enhanced CT and MRI will show enhancement similar to the blood pool. All cases should be treated because of the high risk of perforation.

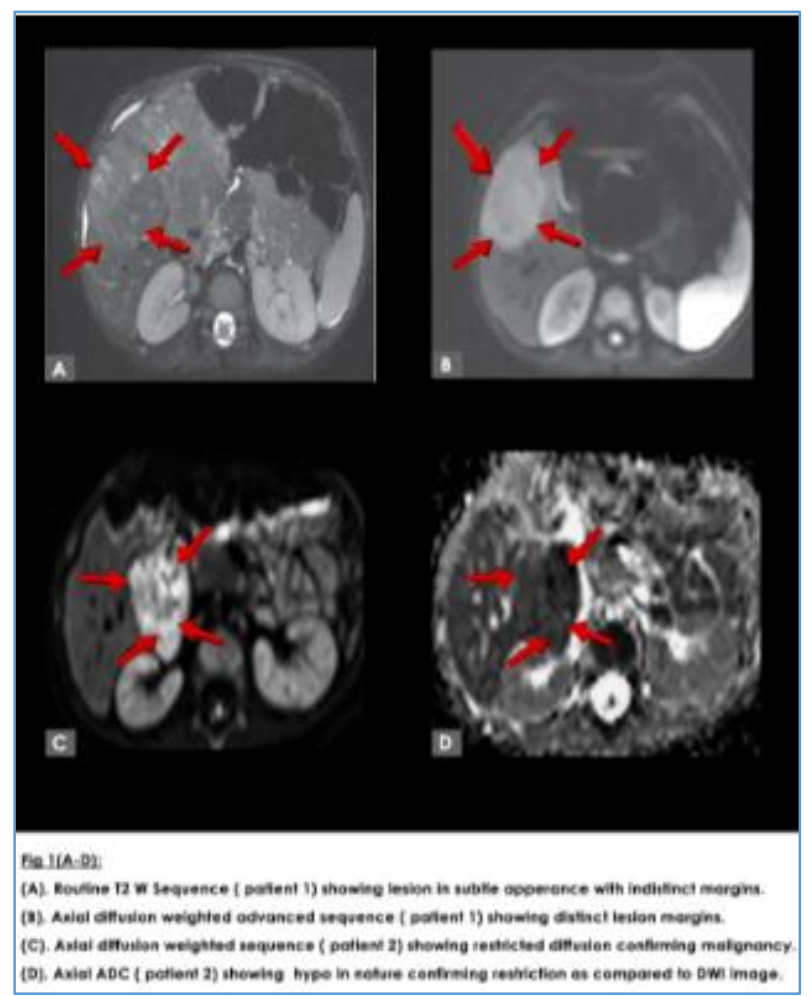

In our sample study, Figure 1 is showing indistinct margins. Routine T2 and T1 weighted sequences are better delineated with diffusion sequence. Routine protocols done in hepatobiliary imaging are $\mathrm{T} 1 \mathrm{~W}$ and $\mathrm{T} 2 \mathrm{~W}$ sequences in axial, sagittal and coronal planes. Modified protocols along with routine of significance are fat saturation sequence, diffusion and ADC sequence; and if vascular lesions were suspected angiography $2 \mathrm{D}$ or $3 \mathrm{D}$ TOF sequence was required. In our study, one case of portal pseudoaneurysm was seen as mentioned in Figure 2. Likewise, Figure 2 showing large hepatic cyst in right lobe of liver turned out to be portal vein aneurysm done by MR angiography.

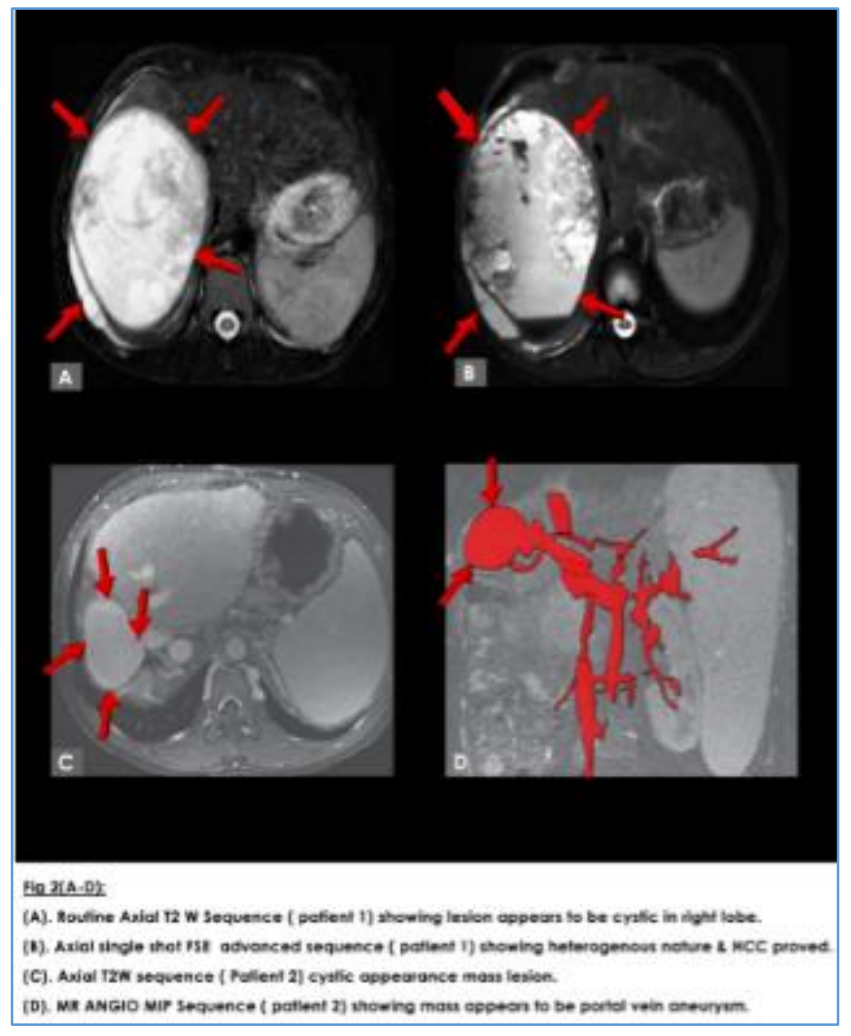

\section{CONCLUSION}

MR modified protocol approach for solitary cystic liver lesions proves to have better specificity as compared to standard protocol. In future, prospective clinician should be aware of modified protocol for specific liver pathology for better treatment planning and optimal detection of pathology.

\section{ACKNOWLEDGEMENT}

We thank Venkatesan Zigma, PhD, for the help with statistical analysis.

\section{REFERENCES}

[1] Semelka RC, Shoenut JP, Kroeker MA, et al. Focal liver disease: comparison of dynamic contrast-enhanced CT and T2-weighted fat-suppressed, FLASH, and dynamic gadolinium-enhanced MR imaging at $1.5 \mathrm{~T}$. Radiology 1992;184(3):687-94.

[2] Weinreb JC, Cohen JM, Armstrong E, et al. Imaging the pediatric liver: MRI and CT. AJR 1986;147(4):785-90. 


\section{Jemds.com}

[3] Roebuck DJ, Olsen O, Pariente D. Radiological staging in children with hepatoblastoma. Pediatr Radiol 2006;36(3):176-82.

[4] Roebuck DJ, Aronson D, Clapuyt P, et al. 2005 PRETEXT: a revised staging system for primary malignant liver tumours of childhood developed by the SIOPEL group. Pediatr Radiol 2007;37(2):123-32; quiz, 249-50.

[5] Arcement CM, Meza MP, Arumanla S, et al. MRCP in the evaluation of pancreaticobiliary disease in children. Pediatr Radiol 2001;31(2):92-7.

\section{Original Research Article}

[6] Delaney L, Applegate KE, Karmazyn B, et al. MR cholangiopancreatography in children: feasibility, safety, and initial experience. Pediatr Radiol 2008;38(1):64-75.

[7] Seale MK, Catalano OA, Saini S, et al. Hepatobiliaryspecific MR contrast agents: role in imaging the liver and biliary tree. Radio Graphics 2009;29(6):1725-48.

[8] Schneider G, Schürholz H, Kirchin MA, et al. Safety and adverse effects during 24 hours after contrastenhanced MRI with gadobenate dimeglumine (MultiHance) in children. Pediatr Radiol 2013;43(2):202-11. 